Moriya, K

Osaka J. Math.

38 (2001), 271-285

\title{
ON A MODULI SPACE OF MINIMAL ANNULI
}

\author{
KATSUHIRO MORIYA
}

(Received May 12, 1999)

\section{Introduction}

The purpose of this paper is to discuss moduli spaces of certain minimal surfaces and their geometric properties.

A complete conformal minimal immersion $X$ from an open Riemann surface $M$ to Euclidean space $\mathbb{R}^{n}$ of finite total curvature is an immersion such that $M$ can be compactified conformally. Moduli spaces of these minimal immersions have been studied from several viewpoints (cf. J. Pérez and A. Ros [8, 9], A. Ros [12], K. Yang [13], R. Kusner and N. Schmitt [4], G. P. Pirola [10, 11], and K. Moriya [5]).

The Riemann surface $M$ can also be compactified conformally in the case where $X: M \rightarrow \mathbb{R}^{3} / T(v)$ is a branched complete conformal minimal immersion of finite total curvature, where $T(v)$ is the discrete group of isometries generated by a translation by $v \in \mathbb{R}^{3}$ (Lemma 2.1). The Euclidean space $\mathbb{R}^{3}$ is considered as $\mathbb{R}^{3} / T((0,0,0)$ ). We will call a branched complete conformal minimal immersion $X: M \rightarrow \mathbb{R}^{3} / T(v)$ of finite total curvature a minimal surface of algebraic type, or simply, an algebraic minimal surface.

Xiaokang Mo studied a moduli space of Weierstrass data for algebraic minimal surfaces in $\mathbb{R}^{3}$ in terms of divisor spaces and Kichoon Yang introduced it in his book [13]. When we consider Weierstrass data, it is natural for us to consider branched minimal immersions.

Mo and Yang obtained a lower bound of the dimension of a complex analytic variety contained in the moduli space. Unfortunately, this is negative in some cases, for example when the genus of the Riemann surface is high. Therefore, we should examine concrete examples of moduli spaces.

We will call an algebraic minimal surface of genus 0 with two puncture points an algebraic minimal annulus. In K. Moriya [6], an example of a moduli space of Weierstrass data for algebraic minimal annuli is investigated in terms of divisor spaces and the defining equations of the moduli space are obtained.

In this paper, we will discuss concrete examples of moduli spaces of Weierstrass data for algebraic minimal annuli in terms of linear systems.

Let us denote by $P$ a pair of integers $\left(P_{0}, P_{\infty}\right)$ which belongs to the set $I:=$ $\{(3,3),(2,3),(3,2),(1,3),(2,2),(3,1)\}$. We will denote by $\mathcal{W}(P, v)$ the set of Weierstrass data for algebraic minimal annuli from $\mathbb{C}-\{0\}$ to $\mathbb{R}^{3} / T(v)$ satisfying the fol- 
lowing conditions:

1. The Gauss map $g(z)=z$.

2. The order of the puncture point 0 and that of the puncture point $\infty$ are equal to $P_{0}$ and $P_{\infty}$ respectively.

We will define the sets of Weierstrass data $\mathcal{W}(v)$ and $\mathcal{W}$ by

$$
\mathcal{W}(v):=\bigcup_{P \in I} \mathcal{W}(P, v), \quad \mathcal{W}:=\bigcup_{P \in I} \bigcup_{v \in \mathbb{R}^{3}} \mathcal{W}(P, v) .
$$

Our main result is that $\mathcal{W}$ has a structure of a 6-dimensional smooth manifold with a Hermitian metric satisfying the following conditions:

1. The scalar curvature is a positive constant.

2. Each $\mathcal{W}(v)\left(v \in \mathbb{R}^{3}\right)$ is a 3-dimensional totally real submanifold in $\mathcal{W}$.

3. A point in $\mathcal{W}$ is a helicoid if and only if it is a point in $\mathcal{W}\left(\left(0,0, v_{3}\right)\right)\left(v_{3} \neq 0\right)$ which attains the maximal value of the scalar curvature of $\mathcal{W}\left(\left(0,0, v_{3}\right)\right)$ with respect to the metric induced from the Hermitian metric on $\mathcal{W}$.

4. The curve in $\mathcal{W}$ which corresponds to an associated family of a minimal annulus in $\mathcal{W}$ is a geodesic.

The author would like to express his gratitude to Y. Ohnita for calling my attention to the study of geometric structures on moduli spaces.

\section{A classification of Weierstrass data}

In this section, we will overview the minimal surface theory briefly and classify Weierstrass data for algebraic minimal annuli by the orders of the branch points and those of the puncture points. For more details, see D. Hoffman and H. Karcher [3], K. Moriya [5, 6], and K. Yang [13].

Let $X: M \rightarrow \mathbb{R}^{3} / T(v)$ be a branched complete conformal minimal immersion. When $v \neq(0,0,0)$, we will assume that there exists a branched complete conformal minimal immersion $\tilde{X}: \tilde{M} \rightarrow \mathbb{R}^{3}$ such that $\Pi \circ \tilde{X}=X \circ \pi$, where $\pi: \tilde{M} \rightarrow M$ is a holomorphic covering, $\tilde{M}$ connected, and $\Pi: \mathbb{R}^{3} \rightarrow \mathbb{R}^{3} / T(v)$ the natural covering. We will identify $X$ with $\tilde{X}$. In the case where $X$ is unbranched, the assumption of the existence of $\tilde{X}$ is not necessary since there exists an immersion $\tilde{X}$ by the half space theorem for minimal surfaces (D. Hoffman and W. H. Meeks, III [2]). In the case where $v=(0,0,0)$, we can assume that $\tilde{X}=X$.

Next, we will modify the Chern-Osserman theorem (S. Chern and R. Osserman [1]).

Let $X: M \rightarrow \mathbb{R}^{3} / T(v)$ be a branched complete conformal minimal immersion. We will denote by $\Phi=\left(\Phi_{1}, \Phi_{2}, \Phi_{3}\right)$ the triplet of holomorphic functions on $M$ such that

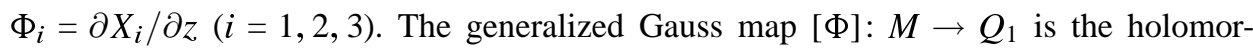
phic map defined by

$$
[\Phi](p)=\left[\Phi_{1}(p), \Phi_{2}(p), \Phi_{3}(p)\right]
$$


where $z$ is a local holomorphic coordinate on $M,\left[z_{1}, z_{2}, z_{3}\right]$ is the homogeneous coordinates of $\mathbb{C} P^{2}$, and $Q_{1}$ is the complex hyperquadric in $\mathbb{C} P^{2}$ :

$$
Q_{1}=\left\{\left[z_{1}, z_{2}, z_{3}\right] \in \mathbb{C} P^{2} \mid\left(z_{1}\right)^{2}+\left(z_{2}\right)^{2}+\left(z_{3}\right)^{2}=0\right\} .
$$

For a triplet $\zeta=\left(\zeta_{1}, \zeta_{2}, \zeta_{3}\right)$ of meromorphic one-forms on a compact Riemann surface $\bar{M}$, we will define the divisor $(\zeta)$ of $\zeta$ by

$$
(\zeta):=\sum_{p \in \bar{M}}\left(\min _{i=1,2,3} \operatorname{ord}_{p} \zeta_{i}\right) \cdot p,
$$

where $\operatorname{ord}_{p} \zeta_{i}$ is the order of $\zeta_{i}$ at $p(i=1,2,3)$.

For a divisor $D$ on $\bar{M}$, we will denote by $\operatorname{mult}_{p} D$ the multiplicity of $D$ at $p$ and by $\operatorname{supp} D$ the support of $D$ :

$$
\operatorname{supp} D:=\left\{p \in \bar{M} \mid \text { mult }_{p} D \neq 0\right\} \text {. }
$$

Let us define two nonnegative divisors $D_{+}$and $D_{-}$on $\bar{M}$ by

$$
\begin{aligned}
& D_{+}:=\sum_{p \in \bar{M}} \max \left\{\operatorname{mult}_{p} D, 0\right\} \cdot p, \\
& D_{-}:=\sum_{p \in \bar{M}} \max \left\{-\operatorname{mult}_{p} D, 0\right\} \cdot p .
\end{aligned}
$$

Then, we can see that $D=D_{+}-D_{-}$.

We will denote by $\Psi_{i}$ the holomorphic one-form on $M$ defined by $\Psi_{i}$ := $\left(\partial X_{i} / \partial z\right) d z(i=1,2,3)$, where $z$ is a holomorphic coordinate on $M$. We will modify the Chern-Osserman theorem as follows:

Lemma 2.1. Let $X: M \rightarrow \mathbb{R}^{3} / T(v)$ be a branched conformal minimal immersion. Then the following conditions are equivalent:

(i) The Riemann surface $M$ is a certain compact Riemann surface $\bar{M}$ with finitely many points $\left\{p_{1}, \ldots, p_{r}\right\}$ removed, $M$ is complete, and $X$ is of finite total curvature. (ii) There exist a compact Riemann surface $\bar{M}$ and a triplet $\bar{\Psi}=\left(\bar{\Psi}_{1}, \bar{\Psi}_{2}, \bar{\Psi}_{3}\right)$ of meromorphic one-forms on $\bar{M}$ such that $\operatorname{supp}(\bar{\Psi})_{-}=\left\{p_{1}, \ldots, p_{r}\right\}$ and that $\left.\bar{\Psi}_{i}\right|_{M}=\Psi_{i}$ $(i=1,2,3)$.

Proof. Assume (i). Then, we can show that (ii) is satisfied in a similar fashion to the proof of the Chern-Osserman theorem and its generalization (cf. [5]) since the generalized Gauss map of $X$ is well-defined.

Conversely, assume (ii). Let $z$ be a local holomorphic coordinate centered at a puncture point and $d s^{2}$ the induced metric. Then, 


$$
d s^{2}=\left(\frac{h(z)}{|z|^{2 m}}\right)|d z|^{2},
$$

where $m$ is a positive integer and $h(z)$ a nonnegative smooth function such that $h(0) \neq$ 0 . Hence, (i) is satisfied.

We will call a point $p_{i}$ a puncture point of $X(i=1, \ldots, r)$. Assume that $(\bar{\Psi})=$ $\sum_{j=1}^{l} B_{j} \cdot b_{j}-\sum_{i=1}^{r} P_{i} \cdot p_{i}\left(B_{j}>0, P_{i}>0\right)$. We can see that the set of points $\left\{b_{1}, \ldots, b_{l}\right\}$ coincides with the set of branch points of $X$.

Definition 2.2. We will call $B_{j}$ the order of a branch point $b_{j}(j=1, \ldots, l)$ and $P_{i}$ the order of a puncture point $p_{i}(i=1, \ldots, r)$.

Definition 2.3. We will call $\tilde{B}:=\sum_{j=1}^{l} B_{j}$ the total order of branch points of $X$ and $\tilde{P}:=\sum_{i=1}^{r} P_{i}$ the total order of puncture points of $X$.

For an immersion $X: M \rightarrow \mathbb{R}^{3} / T(v)$, let us denote by $\tau(X)$ the total curvature of $X$.

Lemma 2.4. If $X: M \rightarrow \mathbb{R}^{3} / T(v)$ is an algebraic minimal surface, then

$$
\tau(X)=2 \pi(\chi(\bar{M})+\tilde{B}-\tilde{P}),
$$

where $\bar{M}$ is the compactified Riemann surface from $M$ and $\chi(\bar{M})$ is the Euler number of $\bar{M}$.

Proof. In the case where $v=(0,0,0)$, Lemma 2.4 was proved by the author ([5, Corollary 2.2]).

Since $\chi(\bar{M}), \tilde{B}$, and $\tilde{P}$ are independent of $v$, the relation (2.1) holds in the case where $v \neq(0,0,0)$.

Since $X$ is conformal, the relation

$$
\left(\bar{\Psi}_{1} \otimes \bar{\Psi}_{1}\right)+\left(\bar{\Psi}_{2} \otimes \bar{\Psi}_{2}\right)+\left(\bar{\Psi}_{3} \otimes \bar{\Psi}_{3}\right)=0
$$

holds on $\bar{M}$ except at the puncture points. We will call this condition the conformality condition of $\bar{\Psi}$.

Assume that $\bar{M}=\mathbb{C} P^{1}=\mathbb{C} \cup\{\infty\}$ and the set of puncture points is $\{0, \infty\}$. Then, we may assume that

$$
(\bar{\Psi})=\sum_{j=1}^{l} B_{j} \cdot b_{j}-P_{0} \cdot 0-P_{\infty} \cdot \infty,
$$


where $B_{j}(j=1, \ldots, l), P_{0}$ and $P_{\infty}>0$. We will call the condition (2.3) the divisor condition of $\bar{\Psi}$.

Since $\bar{\Psi}$ corresponds to an algebraic minimal annulus $X: \mathbb{C}-\{0\} \rightarrow \mathbb{R}^{3} / T(v)$, we may assume that the relation

$$
-2 \pi \operatorname{Im} \operatorname{Res}\left(\bar{\Psi}_{i} ; 0\right)=v_{i}
$$

holds $(i=1,2,3)$, where $\operatorname{Res}\left(\bar{\Psi}_{i} ; 0\right)$ is the residue of $\bar{\Psi}_{i}$ at 0 . We will call the condition (2.4) the period condition of $\bar{\Psi}$.

Conversely, if there exists a triplet $\bar{\Psi}=\left(\bar{\Psi}_{1}, \bar{\Psi}_{2}, \bar{\Psi}_{3}\right)$ of meromorphic one-forms on $\mathbb{C} P^{1}$ satisfying the conformality condition (2.2), the divisor condition (2.3), and the period condition (2.4), then we can obtain an algebraic minimal annulus $X: \mathbb{C}-\{0\} \rightarrow$ $\mathbb{R}^{3} / T(v)$ by integration

$$
X(z):=\operatorname{Re} \int_{z_{0}}^{z} \bar{\Psi}
$$

where $z_{0} \in \mathbb{C}-\{0\}$. If we choose another base point in the integral, the image of the map shifts by a translation in $\mathbb{R}^{3}$.

We can see that a triplet $\bar{\Psi}=\left(\bar{\Psi}_{1}, \bar{\Psi}_{2}, \bar{\Psi}_{3}\right)$ of meromorphic one-forms on $\mathbb{C} P^{1}$ satisfying the conformality condition (2.2) and the divisor condition (2.3) is equivalent to a pair $(g, \eta)$ consisting of a meromorphic function $g$ on $\mathbb{C} P^{1}$ and a meromorphic one-form $\eta$ on $\mathbb{C} P^{1}$ satisfying the following condition:

$$
-(g)_{+}-(g)_{-}+(\eta)=\sum_{j=1}^{l} B_{j} \cdot b_{j}-P_{0} \cdot 0-P_{\infty} \cdot \infty,
$$

where $(g)$ is the divisor of $g$. The following relations hold between $\bar{\Psi}$ and $(g, \eta)$ :

$$
\begin{aligned}
(g, \eta) & =\left(\frac{\bar{\Psi}_{3}}{\bar{\Psi}_{1}-\sqrt{-1} \bar{\Psi}_{2}}, \bar{\Psi}_{3}\right), \\
\left(\bar{\Psi}_{1}, \bar{\Psi}_{2}, \bar{\Psi}_{3}\right) & =\left(\frac{1}{g}-g, \sqrt{-1}\left(\frac{1}{g}+g\right), 2\right) \frac{\eta}{2} .
\end{aligned}
$$

Hence, the following relation holds among $(\bar{\Psi}),(g)$, and $(\eta)$ :

$$
(\bar{\Psi})=-(g)_{+}-(g)_{-}+(\eta)
$$

Thus, a pair $(g, \eta)$ corresponds to an algebraic minimal annuli if and only if $(g, \eta)$ satisfies the condition (2.5) and the triplet $\bar{\Psi}$ of meromorphic one-forms on $\mathbb{C} P^{1}$ equivalent to $(g, \eta)$ satisfies the period condition (2.4). We will call the pair $(g, \eta)$ associated to an algebraic minimal annulus $X: \mathbb{C}-\{0\} \rightarrow \mathbb{R}^{3} / T(v)$ Weierstrass data of $X$. 
Let $N: \mathbb{C}-\{0\} \rightarrow S^{2}$ be the normal Gauss map of $X$ and $\pi: S^{2}-\{(0,0,1)\} \rightarrow \mathbb{R}^{2}$ the stereographic projection, where $S^{2}$ is a 2-dimensional sphere. Then, we can see that $\left.g\right|_{\mathbb{C}-\{0\}}=\pi \circ N$.

Let us assume that the total curvature of a minimal annulus corresponding to $(g, \eta)$ is equal to $-4 \pi$. Then, we can obtain the following relation by $(2.1)$ :

$$
\tilde{B}-\tilde{P}=-4 .
$$

Since $\eta$ is a meromorphic one-form on $\mathbb{C} P^{1}$, the degree of $(\eta)$ is equal to -2 . Hence, we can see that the relation

$$
\tilde{B}-\tilde{P}=-2 \operatorname{deg} g-2
$$

holds by (2.5). Thus, we can see that $\operatorname{deg} g=1$ by the relation (2.7).

Let us denote by $z$ a holomorphic coordinate of $\mathbb{C}$ centered at the origin. If we take another coordinate $w=1 / z$, the point 0 and $\infty$ are exchanged each other in the divisor condition (2.5). Hence, the following three cases are sufficient for us to investigate minimal annuli:

- $(g)=1 \cdot 0-1 \cdot \infty$.

- $\quad(g)=1 \cdot a-1 \cdot \infty, a \neq 0$.

- $\quad(g)=1 \cdot a-1 \cdot b, a \neq 0, b \neq \infty$.

In the following, we will consider only the first case. Then, we can see that $g=a z$ $\left(a \in \mathbb{C}^{*}\right)$ and hence

$$
(\eta)=\sum_{j=1}^{k} B_{j} \cdot b_{j}-\left(P_{0}-1\right) \cdot 0-\left(P_{\infty}-1\right) \cdot \infty,
$$

from the divisor condition (2.5).

By (2.8), we can see that $\left(P_{0}, P_{\infty}\right) \in I$ if and only if

$$
\eta=\frac{c_{0} z^{2}+\left(c_{1} / \sqrt{2}\right) z+c_{2}}{z^{2}} d z
$$

where $I$ is the set of pairs of integers defined in Section 1 and $\left(c_{0}, c_{1}, c_{2}\right) \in \mathbb{C}^{3}-\{0\}$. By (2.6), we can see that $0 \leq \tilde{B} \leq 2$ if $\left(P_{0}, P_{\infty}\right) \in I$.

The Weierstrass data

$$
\left(a z, \frac{a c_{0} z^{2}+\left(c_{1} / \sqrt{2}\right) z+c_{2} / a}{z^{2}} d z\right)
$$

and

$$
\left(z, \frac{c_{0} z^{2}+\left(c_{1} / \sqrt{2}\right) z+c_{2}}{z^{2}} d z\right)
$$




\begin{tabular}{c|c||c||c|c|c}
$P_{0}$ & $P_{\infty}$ & $\tilde{B}$ & $c_{0}$ & $c_{1}$ & $c_{2}$ \\
\hline 3 & 3 & 2 & $\neq 0$ & $*$ & $\neq 0$ \\
2 & 3 & 1 & $\neq 0$ & $\neq 0$ & 0 \\
3 & 2 & 1 & 0 & $\neq 0$ & $\neq 0$ \\
1 & 3 & 0 & $\neq 0$ & 0 & 0 \\
2 & 2 & 0 & 0 & $\neq 0$ & 0 \\
3 & 1 & 0 & 0 & 0 & $\neq 0$
\end{tabular}

Table 1. the relation among $\left(P_{0}, P_{\infty}\right), \tilde{B}$, and $\left(c_{0}, c_{1}, c_{2}\right)$

produce the same algebraic minimal annulus since these data are obtained by rescaling the holomorphic coordinate $z$ on $\mathbb{C}$ each other. Hence, we will consider only the latter case in the following.

Let $\mathcal{W}(P, v), \mathcal{W}(v)$, and $\mathcal{W}$ be the sets of Weierstrass data defined in Section 1 . We will denote by $\mathcal{A}(P, v)$ the set of algebraic minimal annuli whose Weierstrass data belong to $\mathcal{W}(P, v)$. The notation $X \sim Y$ for $X$ and $Y$ in $\mathcal{A}(P, v)$ means that $X+x=$ $Y$ for some $x \in \mathbb{R}^{3}$. Summarizing the above discussion, we can prove the following lemmas:

Lemma 2.5. There exists a bijective correspondence between the set $\mathcal{A}(P, v) / \sim$ and the set $\mathcal{W}(P, v)$.

Lemma 2.6. The set $\mathcal{W}$ is equal to the set

$$
\left\{\left(z, \frac{c_{0} z^{2}+\left(c_{1} / \sqrt{2}\right) z+c_{2}}{z^{2}} d z\right) \mid\left(c_{0}, c_{1}, c_{2}\right) \in \mathbb{C}^{3}-\{0\}\right\} .
$$

Lemma 2.7. The set $\mathcal{W}(v)$ consists of each element $(z, \eta)$ of $\mathcal{W}$ satisfying the following conditions:

$$
\begin{aligned}
& -2 \pi \operatorname{Im} \operatorname{Res}\left(\left(\frac{1}{z}-z\right) \frac{\eta}{2} ; 0\right)=v_{1}, \\
& -2 \pi \operatorname{Im} \operatorname{Res}\left(\sqrt{-1}\left(\frac{1}{z}+z\right) \frac{\eta}{2} ; 0\right)=v_{2}, \\
& -2 \pi \operatorname{Im} \operatorname{Res}(\eta ; 0)=v_{3} .
\end{aligned}
$$

Lemma 2.8. The relations among the total order of branch points $\tilde{B}$, the order of each puncture point $P_{0}$ and $P_{\infty}$, and the values of $c_{0}, c_{1}$, and $c_{2}$ become as Table 1 , where $*$ means any complex number.

We will consider the set $S^{1}:=\{\exp [\sqrt{-1} t] \mid t \in \mathbb{R}\}$ as a Lie group. Let $\mu: \mathcal{W} \times$ $S^{1} \rightarrow \mathcal{W}$ be the map defined by 


$$
\mu((z, \eta), \exp [\sqrt{-1} t])=(z,(\exp [\sqrt{-1} t]) \eta)
$$

Then, $\mu$ is an action of $S^{1}$ on $\mathcal{W}$. This action is transitive and effective. The orbit of an element $(z, \eta) \in \mathcal{W}$ is the set of Weierstrass data for the associated family of a minimal annulus produced by $\eta$.

Definition 2.9. We will call the orbit of $(z, \eta) \in \mathcal{W}$ by $\mu$ the associated orbit of $(z, \eta)$

We will recall that an unbranched complete minimal annulus in $\mathbb{R}^{3}$ with total curvature $-4 \pi$ is a catenoid (Osserman [7]). The Weierstrass data for a catenoid is given by $(z, r d z / z)(r \in \mathbb{R}, r \neq 0)$. Hence, the order of each puncture point is equal to 2 . Since a helicoid is the conjugate surface of a catenoid, the Weierstrass data for a helicoid is given by $(z, \sqrt{-1} s d z / z)(s \in \mathbb{R}, s \neq 0)$. Hence, the order of each puncture point is equal to 2 , too. Since we obtain

$$
\begin{aligned}
& -2 \pi \operatorname{Im} \operatorname{Res}\left(\left(\frac{1}{z}-z\right) \frac{\sqrt{-1} s d z}{2 z} ; 0\right)=0, \\
& -2 \pi \operatorname{Im} \operatorname{Res}\left(\sqrt{-1}\left(\frac{1}{z}+z\right) \frac{\sqrt{-1} s d z}{2 z} ; 0\right)=0, \\
& -2 \pi \operatorname{Im} \operatorname{Res}\left(\frac{\sqrt{-1} s d z}{z} ; 0\right)=-s,
\end{aligned}
$$

we can see that the Weierstrass data $(z, \sqrt{-1} s d z / z)(s \in \mathbb{R}, s \neq 0)$ belongs to $\mathcal{W}((2,2),(0,0,-s))$ by $(2.9)$. Thus, the Weierstrass data $(z, \eta)$ of the catenoid and of the helicoid belong to $\mathcal{W}((2,2),(0,0,0))$ and $\mathcal{W}\left((2,2),\left(0,0, v_{3}\right)\right)\left(v_{3} \neq 0\right)$ respectively. We can see that the Weierstrass data $(z,(r+\sqrt{-1} s) d z / z)(r s \neq 0)$ produces a minimal annulus which is neither a catenoid nor a helicoid.

By the above discussion, we have proved the following lemmas:

Lemma 2.10. A point $(z, \eta) \in \mathcal{W}((0,0,0))$ corresponds to a catenoid if and only if $\eta=r d z / z(r \in \mathbb{R}, r \neq 0)$.

Lemma 2.11. A point $(z, \eta) \in \mathcal{W}\left(\left(0,0, v_{3}\right)\right)\left(v_{3} \neq 0\right)$ corresponds to a helicoid if and only if $\eta=-\sqrt{-1} v_{3} d z / z$.

\section{A submanifold of Weierstrass data}

In this section, we will show two kinds of moduli spaces denoted by $\mathcal{W}(v)$ and $\mathcal{W}(P)$ become smooth submanifolds in $\mathcal{W}$.

We can consider $\mathcal{W}$ as $\mathbb{C}^{3}-\{0\}$ with holomorphic coordinates $\left(c_{0}, c_{1}, c_{2}\right)$. Let us denote by $J_{0}$ the complex structure. We will define real coordinates $\left(u_{1}, \ldots, u_{6}\right)$ on $\mathcal{W}$ 
by $u_{1}:=\operatorname{Re} c_{0}, u_{2}:=\operatorname{Im} c_{0}, u_{3}:=\operatorname{Re} c_{1}, u_{4}:=\operatorname{Im} c_{1}, u_{5}:=\operatorname{Re} c_{2}$, and $u_{6}:=\operatorname{Im} c_{2}$.

Theorem 3.1. The set $\mathcal{W}(v)$ is a 3-dimensional connected real algebraic smooth submanifold of $\mathcal{W}$ defined by

$$
\mathcal{W}(v)=\left\{\left(u_{1}, u_{2}, u_{3},-\sqrt{2} v_{3},-u_{1}-2 v_{2}, u_{2}+2 v_{1}\right) \in \mathcal{W}\right\}
$$

Proof. The set $\mathcal{W}(v)$ consists of each element $\left(u_{1}, \ldots, u_{6}\right) \in \mathbb{R}^{6}-\{0\}$ satisfying the following equations:

$$
-\frac{1}{2}\left(u_{2}-u_{6}\right)=v_{1},-\frac{1}{2}\left(u_{1}+u_{5}\right)=v_{2},-\frac{1}{\sqrt{2}} u_{4}=v_{3} .
$$

Simplifying the equations (3.2), we can obtain the following equations:

$$
u_{4}=-\sqrt{2} v_{3}, \quad u_{5}=-u_{1}-2 v_{2}, u_{6}=u_{2}+2 v_{1} .
$$

Thus, the set $\mathcal{W}(v)$ is obtained by (3.1).

Let $\mathcal{W}(P)$ be the subset of $\mathcal{W}$ defined by $\mathcal{W}(P):=\bigcup_{v \in \mathbb{R}^{3}} \mathcal{W}(P, v)$, where $P=$ $\left(P_{0}, P_{\infty}\right) \in I$.

Theorem 3.2. The set $\mathcal{W}(P)$ is a $(\tilde{B}+1)$-dimensional algebraic complex submanifold of $\left(\mathcal{W}, J_{0}\right)$ where $\tilde{B}$ is the total order of branch points corresponding to $P$ as in Lemma 2.8.

Proof. We will prove this theorem in the case where $P=(2,2)$. In the other cases, we can prove the theorem in a similar fashion.

The set $\mathcal{W}(P)$ is the set of solutions of the equations $u_{1}+\sqrt{-1} u_{2}=0$ and $u_{5}+$ $\sqrt{-1} u_{6}=0$ on $\mathcal{W}-\left\{u_{3}+\sqrt{-1} u_{4} \neq 0\right\}$. The total order of branch points of a minimal annulus which corresponds to an element of $\mathcal{W}(P)$ is equal to 0 by Table 1 . Since $u_{1}+$ $\sqrt{-1} u_{2}$ and $u_{5}+\sqrt{-1} u_{6}$ are holomorphic, we can see that $\mathcal{W}(P)$ is a one-dimensional algebraic complex submanifold of $\left(\mathcal{W}, J_{0}\right)$.

\section{Geometry of moduli spaces}

In this section, we will investigate geometric properties of moduli spaces $\mathcal{W}$, $\mathcal{W}(v)$, and $\mathcal{W}(P)$. We will show that each moduli space $\mathcal{W}(v)\left(v \in \mathbb{R}^{3}\right)$ becomes a 3-dimensional totally real submanifold of the 6-dimensional manifold $\mathcal{W}$.

Firstly, we will investigate the geometry of $\mathcal{W}$.

Let $\rho=\left(\rho_{1}, \ldots, \rho_{7}\right): \mathcal{W} \rightarrow \mathbb{R}^{7}$ be an immersion such that

$$
\rho_{\alpha}\left(u_{1}, \ldots, u_{6}\right)=\frac{u_{\alpha}}{u}(\alpha=1, \ldots, 6), \rho_{7}\left(u_{1}, \ldots, u_{6}\right)=\log u,
$$


where $u=\left(\sum_{\alpha=1}^{6} u_{\alpha}^{2}\right)^{1 / 2}$. Then, $\rho(\mathcal{W})$ is a high-dimensional cylinder, that is $\rho(\mathcal{W})=$ $S^{5} \times \mathbb{R}$, where $S^{5}$ is a 5 -dimensional sphere.

Let $g_{0}$ be the metric on $\mathcal{W}$ induced from the standard Riemannian metric on $\mathbb{R}^{7}$ by $\rho$. Then,

$$
g_{0}=\frac{1}{u^{2}}\left(\sum_{\alpha=1}^{6} d u_{\alpha}^{2}\right)
$$

Proposition 4.1. The scalar curvature of $\left(\mathcal{W}, g_{0}\right)$ is equal to 20.

Proof. Let us denote by $\left(\theta^{1}, \ldots, \theta^{6}\right)$ an orthonormal coframe of $\left(\mathcal{W}, g_{0}\right)$, where $\theta^{\alpha}:=d u_{\alpha} / u(\alpha=1, \ldots, 6)$. Then, $g_{0}=\sum_{\alpha=1}^{6} \theta^{\alpha} \otimes \theta^{\alpha}$. Let us denote the first and the second structure equations of the Levi-Civita connection by

$$
d \theta^{\alpha}=-\sum_{\beta=1}^{6} \omega_{\beta}{ }^{\alpha} \wedge \theta^{\beta}, \Omega_{\beta}{ }^{\alpha}=d \omega_{\beta}{ }^{\alpha}+\sum_{\gamma=1}^{6} \omega_{\gamma}{ }^{\alpha} \wedge \omega_{\beta}{ }^{\gamma},
$$

where $\omega_{\beta}{ }^{\alpha}$ is the connection form and $\Omega_{\beta}{ }^{\alpha}$ is the curvature form $(\alpha, \beta=1, \ldots, 6)$. We can see that a connection form $\omega_{\beta}{ }^{\alpha}$ becomes

$$
\omega_{\beta}^{\alpha}=-\frac{u_{\beta}}{u} \theta^{\alpha}+\frac{u_{\alpha}}{u} \theta^{\beta}
$$

and a curvature form $\Omega_{\beta}{ }^{\alpha}:=(1 / 2) \sum_{\gamma, \delta=1}^{6} R^{\alpha}{ }_{\beta \gamma \delta} \theta^{\gamma} \wedge \theta^{\delta}$ becomes

$$
\begin{aligned}
\Omega_{\beta}{ }^{\alpha}= & \left(1-\frac{u_{\alpha}{ }^{2}+u_{\beta}{ }^{2}}{u^{2}}\right) \theta^{\alpha} \wedge \theta^{\beta} \\
& -\sum_{\gamma=1, \gamma \neq \beta}^{6} \frac{u_{\beta} u_{\gamma}}{u^{2}} \theta^{\alpha} \wedge \theta^{\gamma}+\sum_{\gamma=1, \gamma \neq \alpha}^{6} \frac{u_{\alpha} u_{\gamma}}{u^{2}} \theta^{\beta} \wedge \theta^{\gamma} .
\end{aligned}
$$

Hence, the Ricci curvature Ric : $=\sum_{\beta, \gamma=1}^{6} R_{\beta \gamma} \theta^{\beta} \otimes \theta^{\gamma}$ becomes

$$
\text { Ric }=\sum_{\alpha=1}^{6} 4\left(1-\frac{u_{\alpha}{ }^{2}}{u^{2}}\right) \theta^{\alpha} \otimes \theta^{\alpha}-\sum_{\beta, \gamma=1, \beta \neq \gamma}^{6} \frac{4 u_{\beta} u_{\gamma}}{u^{2}} \theta^{\beta} \otimes \theta^{\gamma} .
$$

Thus, the scalar curvature $\sum_{\alpha=1}^{6} R_{\alpha \alpha}$ is 20 .

Proposition 4.2. An associated orbit of an element of $\mathcal{W}$ is a geodesic in $\left(\mathcal{W}, g_{0}\right)$.

Proof. The image of an associated orbit by $\rho$ is clearly a geodesic of $\rho(\mathcal{W})$. Thus, an associated orbit is a geodesic. 
Secondly, we will investigate the geometry of $\mathcal{W}(v)$.

We will denote by $g_{1}$ the metric on $\mathcal{W}(v)$ induced from $g_{0}$ by the inclusion map. Then, we obtain

Proposition 4.3. The scalar curvature $\sigma_{1}$ of $\left(\mathcal{W}(v), g_{1}\right)$ is 2 if and only if $v=$ $(0,0,0)$.

Proof. Let us take another orthonormal coframe $\left(\tilde{\theta}^{1}, \ldots, \tilde{\theta}^{6}\right)$ of $\left(\mathcal{W}, g_{0}\right)$ defined by the following:

$$
\begin{aligned}
& \tilde{\theta}^{1}=\frac{\theta^{1}-\theta^{5}}{\sqrt{2}}, \quad \tilde{\theta}^{2}=\frac{\theta^{2}+\theta^{6}}{\sqrt{2}}, \quad \tilde{\theta}^{3}=\theta^{3}, \\
& \tilde{\theta}^{4}=\frac{\theta^{2}-\theta^{6}}{\sqrt{2}}, \quad \tilde{\theta}^{5}=\frac{\theta^{1}+\theta^{5}}{\sqrt{2}}, \quad \tilde{\theta}^{6}=\theta^{4} .
\end{aligned}
$$

Then, $g_{1}=\sum_{i=1}^{3} \tilde{\vartheta}^{i} \otimes \tilde{\vartheta}^{i}$ where $\tilde{\vartheta}^{i}=\left.\tilde{\theta}^{i}\right|_{\mathcal{W}(v)}(i=1,2,3)$. Let $\tilde{\omega}_{\beta}{ }^{\alpha}(\alpha, \beta=1, \ldots, 6)$ be the connection form of the Levi-Civita connection with respect to $\left(\tilde{\theta}^{1}, \ldots, \tilde{\theta}^{6}\right)$. Then, we obtain

$$
\tilde{\omega}_{j}{ }^{i}{ }_{\mathcal{W}(v)}=-L_{j} \tilde{\vartheta}^{i}+L_{i} \tilde{\vartheta}^{j},\left.\quad \tilde{\omega}_{j}{ }^{3+i}\right|_{\mathcal{W}(v)}=-\frac{\sqrt{2} v_{i}}{U} \tilde{\vartheta}^{j},\left.\quad \tilde{\omega}_{3+j}{ }^{3+i}\right|_{\mathcal{W}(v)}=0
$$

where

$$
\begin{aligned}
& U=\sqrt{2 u_{1}^{2}+2 u_{2}^{2}+u_{3}^{2}+2 v_{3}^{2}+4 u_{1} v_{2}+4 v_{2}^{2}+4 u_{2} v_{1}+4 v_{1}^{2}}, \\
& L_{1}=\frac{\sqrt{2}\left(u_{1}+v_{2}\right)}{U}, L_{2}=\frac{\sqrt{2}\left(u_{2}+v_{1}\right)}{U}, L_{3}=\frac{u_{3}}{U}
\end{aligned}
$$

$(i, j=1,2,3)$. The components $\tilde{R}_{i, j}$ of the Ricci curvature of $\left(\mathcal{W}(v), g_{1}\right)$ with respect to $\left(\tilde{\vartheta}^{1}, \tilde{\vartheta}^{2}, \tilde{\vartheta}^{3}\right)$ are as follows:

$$
\begin{aligned}
& \tilde{R}_{1,1}=\left(2\left(u_{2}+v_{1}\right)^{2}+u_{3}^{2}+8\left(v_{1}^{2}+v_{2}^{2}+v_{3}^{2}\right)\right) / U^{2}, \\
& \tilde{R}_{1,2}=-\left(2\left(u_{1}+v_{2}\right)\left(u_{2}+v_{1}\right)\right) / U^{2}, \\
& \tilde{R}_{1,3}=-\left(\sqrt{2}\left(u_{1}+v_{2}\right) u_{3}\right) / U^{2}, \\
& \tilde{R}_{2,2}=\left(2\left(u_{1}+v_{2}\right)^{2}+u_{3}^{2}+8\left(v_{1}^{2}+v_{2}^{2}+v_{3}^{2}\right)\right) / U^{2}, \\
& \tilde{R}_{2,3}=-\left(\sqrt{2}\left(u_{2}+v_{1}\right) u_{3}\right) / U^{2}, \\
& \tilde{R}_{3,3}=2\left(\left(u_{1}+v_{2}\right)^{2}+\left(u_{2}+v_{1}\right)^{2}+4\left(v_{1}^{2}+v_{2}^{2}+v_{3}^{2}\right)\right) / U^{2} .
\end{aligned}
$$

Hence, the scalar curvature $\sigma_{1}$ of $\left(\mathcal{W}(v), g_{1}\right)$ becomes

$$
\sigma_{1}=2\left(1+10 \frac{v_{1}^{2}+v_{2}^{2}+v_{3}^{2}}{U^{2}}\right) .
$$


Thus, $\sigma_{1}$ is 2 if and only if $v=(0,0,0)$.

The moduli space $\mathcal{W}((0,0,0))$ is characterized as follows:

Proposition 4.4. The submanifold $\mathcal{W}(v)$ of $\left(\mathcal{W}, g_{0}\right)$ is totally geodesic if and only if $v=(0,0,0)$.

Proof. The second fundamental form $A_{1}$ of $\mathcal{W}(v)$ becomes

$$
A_{1}=-\left.\sum_{i=1}^{3} \sum_{j=1}^{3} \frac{\sqrt{2} v_{j}}{U} \tilde{\vartheta}^{i} \otimes \tilde{\vartheta}^{i} \otimes \tilde{e}_{j+3}\right|_{\mathcal{W}(v)},
$$

where $\left(\tilde{e}_{1}, \ldots, \tilde{e}_{6}\right)$ is the dual frame of $\left(\tilde{\theta}^{1}, \ldots, \tilde{\theta}^{6}\right)$. Hence, the submanifold $\mathcal{W}(v)$ of $\left(\mathcal{W}, g_{0}\right)$ is totally geodesic if and only if $v=(0,0,0)$.

A helicoid is characterized as follows:

Theorem 4.5. A point in $\mathcal{W}\left(\left(0,0, v_{3}\right)\right)\left(v_{3} \neq 0\right)$ corresponds to a helicoid if and only if the point attains the maximum value of the scalar curvature $\sigma_{1}$ of $\left(\mathcal{W}\left(\left(0,0, v_{3}\right)\right), g_{1}\right)$.

Proof. By Lemma 2.11, $\left(u_{1}, \ldots, u_{6}\right) \in \mathcal{W}$ corresponds to a helicoid if and only if $\left(u_{1}, \ldots, u_{6}\right)=\left(0,0,0, u_{4}, 0,0\right)\left(u_{4} \neq 0\right)$.

If $u_{4}=-\sqrt{2} v_{3}$, then $U^{2}=2 u_{1}^{2}+2 u_{2}^{2}+u_{3}^{2}+2 v_{3}^{2}$. Thus, a point in $\mathcal{W}\left(\left(0,0, v_{3}\right)\right)$ $\left(v_{3} \neq 0\right)$ corresponds to a helicoid if and only if the point attains the maximum value of the scalar curvature $\sigma_{1}$ of $\left(\mathcal{W}\left(\left(0,0, v_{3}\right)\right), g_{1}\right)$.

Let us denote by $\mathcal{W}_{t}((0,0,0))(t \neq 0)$ the set $\left\{\left(u_{1}, u_{2}, t, 0,-u_{1}, u_{2}\right) \in \mathcal{W}((0,0,0))\right\}$. We can consider the set $\mathcal{W}_{t}((0,0,0))$ as the set of Weierstrass data for elements of $\bigcup_{P \in H} \mathcal{A}(P,(0,0,0))$ except the set $\left\{u_{3}=0\right\} \subset \mathcal{W}$, where $H=\{(2,2),(2,3),(3,2),(3,3)\}$. We will denote by $g_{3}$ the metric on $\mathcal{W}_{t}((0,0,0))$ induced from $\left(\mathcal{W}, g_{0}\right)$ by the inclusion map.

Theorem 4.6. A point in $\mathcal{W}_{t}((0,0,0))(t \neq 0)$ corresponds to a catenoid if and only if the point attains the maximum value of the Gauss curvature of $\left(\mathcal{W}_{t}((0,0,0)), g_{3}\right)$.

Proof. We can see that $g_{3}=\left(2 d u_{1}^{2}+2 d u_{2}^{2}\right) /\left(2 u_{1}^{2}+2 u_{2}^{2}+t^{2}\right)$. Hence, the scalar curvature of $\left(\mathcal{W}_{t}((0,0,0)), g_{3}\right)$ is equal to $\sqrt{2} t^{2} /\left(2 u_{1}^{2}+2 u_{2}^{2}+t^{2}\right)^{2 / 3}$. By Lemma 2.10, a point $\left(u_{1}, u_{2}, t, 0,-u_{1}, u_{2}\right) \in \mathcal{W}_{t}((0,0,0))(t \neq 0)$ corresponds to a catenoid if and only if $u_{1}=u_{2}=0$. Hence, a point in $\mathcal{W}_{t}((0,0,0))(t \neq 0)$ corresponds to a catenoid if and only if the point attains the maximum value of the Gauss curvature of 
$\left(\mathcal{W}_{t}((0,0,0)), g_{3}\right)$

Next, we will define another complex structure on $\mathcal{W}$.

Let $J$ be the tensor field on $\mathcal{W}$ which is an endomorphism of the tangent space $T_{u} \mathcal{W}$ at every point $u$ of $\mathcal{W}$ such that

$$
\begin{array}{lll}
J \tilde{e}_{1}=\tilde{e}_{5}, & J \tilde{e}_{2}=\tilde{e}_{4}, & J \tilde{e}_{3}=\tilde{e}_{6}, \\
J \tilde{e}_{4}=-\tilde{e}_{2}, & J \tilde{e}_{5}=-\tilde{e}_{1}, & J \tilde{e}_{6}=-\tilde{e}_{3},
\end{array}
$$

where $\left(\tilde{e}_{1}, \ldots, \tilde{e}_{6}\right)$ is the dual frame of $\left(\tilde{\theta}^{1}, \ldots, \tilde{\theta}^{6}\right)$ as in the proof of Proposition 4.3.

Lemma 4.7. The tensor field $J$ is an orthogonal integrable complex structure of $\mathcal{W}$.

Proof. Let $J\left(\partial / \partial u_{\alpha}\right)=\sum_{\beta=1}^{6} J_{\alpha}^{\beta}\left(\partial / \partial u_{\beta}\right)$. Then, all nonzero components of $J$ become as follows:

$$
J_{1}^{5}=J_{2}^{4}=J_{3}^{6}=-J_{5}^{1}=-J^{2}{ }_{4}=-J_{6}^{3}=1 .
$$

Then, we may see $J^{2}=-I$, where $I$ denotes the identity transformation of $T_{u} \mathcal{W}$. The torsion tensor field $N$ of $J$ is defined by $N(X, Y)=[J X, J Y]-[X, Y]-J[X, J Y]-$ $J[J X, Y]$ for vector fields $X$ and $Y$ on $\mathcal{W}$. The component $N^{\alpha}{ }_{\beta \gamma}$ of $N$ is as follows:

$$
N_{\beta \gamma}^{\alpha}=\sum_{\delta=1}^{6}\left(J_{\alpha}^{\delta} \partial_{\delta} J_{\gamma}^{\alpha}-J_{\gamma}^{\delta} \partial_{\delta} J_{\beta}^{\alpha}-J_{\delta}^{\alpha} \partial_{\beta} J_{\gamma}^{\delta}+J_{\delta}^{\alpha} \partial_{\gamma} J_{\beta}^{\delta}\right),
$$

where $\partial_{\delta}$ is the partial differentiation $\partial / \partial u_{\delta}(\alpha, \beta, \gamma, \delta=1, \ldots, 6)$. Hence, $N \equiv 0$ clearly. Thus, $J$ is an integral orthogonal complex structure on $\mathcal{W}$.

Remark 4.8. The metric $g_{0}$ is a Hermitian metric on $(\mathcal{W}, J)$. On the other hand, $g_{0}$ is not a Kähler metric. Indeed, the fundamental 2-form $\Phi_{0}$ of $g_{0}$ is

$$
\Phi_{0}=\tilde{\theta}^{1} \wedge \tilde{\theta}^{5}+\tilde{\theta}^{2} \wedge \tilde{\theta}^{4}+\tilde{\theta}^{3} \wedge \tilde{\theta}^{6} .
$$

Hence, the exterior differential of $\Phi_{0}$ becomes

$$
d \Phi_{0}=-2 \sum_{\alpha=1}^{6}\left(\frac{u_{\alpha}}{u} \theta^{\alpha}\right) \wedge \Phi_{0} .
$$

Thus the 2 -form $\Phi_{0}$ is not closed.

By Theorem 3.1 and Lemma 4.7, we can see that $\mathcal{W}(v)$ is a 3-dimensional real submanifold of a 6-dimensional Hermitian manifold $\left(\mathcal{W}, g_{0}, J\right)$. Moreover, we can see the following theorem holds: 
Theorem 4.9. The submanifold $\mathcal{W}(v)$ is a totally real submanifold of $\left(\mathcal{W}, g_{0}, J\right)$.

Proof. We can see that $\left.\tilde{\theta}^{p}\right|_{\mathcal{W}(v)}=0(p=4,5,6)$. Hence, $\left.\Phi_{0}\right|_{\mathcal{W}(v)}=0$. Thus, $\mathcal{W}(v)$ is a totally real smooth submanifold of $\left(\mathcal{W}, g_{0}, J\right)$.

A real submanifold in a Kähler manifold is called Lagrangian if it satisfies the following conditions:

1. The real dimension of the submanifold is equal to half of the real dimension of the ambient Kähler manifold.

2. The submanifold is a totally real submanifold of the ambient Kähler manifold.

It is an interesting problem to find a Kähler metric on $\mathcal{W}$ which makes $\mathcal{W}(v)$ Lagrangian. Finally, we will mention a sufficient condition for a Kähler metric on $\mathcal{W}$ to make $\mathcal{W}(v)$ Lagrangian.

Theorem 4.10. Let $g^{\prime}$ be a Kähler metric on $(\mathcal{W}, J) \cong \mathbb{R}^{6}-\{0\}$ conformal to the standard metric $g$ of $\mathbb{R}^{6}$. If $\left(\mathcal{W}, g^{\prime}, J\right)$ makes $\mathcal{W}(v)$ Lagrangian, then $g^{\prime}$ is homothetic to $g$, that is $g^{\prime}=c g$ where $c$ is a positive constant.

Proof. Let $g^{\prime}=f g$ where $f$ is a positive function. Then the fundamental 2-form $\Phi^{\prime}$ of $g^{\prime}$ is equal to $f \Phi$, where $\Phi$ is the fundamental 2-form of $g$. Since $\Phi^{\prime}$ and $\Phi$ are closed, the relation $d f \wedge \Phi=0$ holds. Hence $d f=0$. Thus $f$ is constant on $\mathcal{W}$. Therefor $g^{\prime}$ is homothetic to $g$.

REMARK 4.11. The metric $c g$ gives a good geometric property to $\mathcal{W}(v)$. However, this metric does not distinguish moduli spaces $\{\mathcal{W}(v)\}$ by their curvature. Hence, it is impossible to characterize a helicoid and a catenoid by the curvature of the moduli space. Thus, we can find that this metric is not so useful to obtain geometric properties of each individual minimal annulus.

Finally, we will investigate the geometry of $\mathcal{W}(P)$. We will denote by $g_{2}$ the metrics on $\mathcal{W}(P)$ induced from $g_{0}$ by the inclusion map.

Proposition 4.12. The submanifold $\mathcal{W}(P)$ is a totally geodesic submanifold of $\left(\mathcal{W}, g_{0}\right)$.

Proof. We will prove this theorem in the case where $P=(2,2)$. We can prove the other cases in a similar fashion.

The induced metric $g_{2}$ becomes

$$
g_{2}=\vartheta^{3} \otimes \vartheta^{3}+\vartheta^{4} \otimes \vartheta^{4}
$$

where $\vartheta^{i}=\left.\theta^{i}\right|_{\mathcal{W}(P)}(i=3,4)$. Hence, $\left.\omega_{i}\right|_{\mathcal{W}(P)}=0(i=3,4, \quad p=1,2,5,6)$. Thus, the 
second fundamental form $A$ of $\mathcal{W}(P)$ vanishes: $A \equiv 0$. Therefore $\mathcal{W}(P)$ is a totally geodesic submanifold of $\mathcal{W}$.

\section{References}

[1] S. Chern and R. Osserman: Complete minimal surfaces in euclidean n-space, J. Analyse Math. 19 (1967), 15-34.

[2] D. Hoffman and W.H. Meeks, III: The strong halfspace theorem for minimal surfaces, Invent. Math. 101 (1990), 373-377.

[3] D. Hoffman and H. Karcher: Complete embedded minimal surfaces of finite total curvature, Geometry, V, Springer, Berlin, 5-93, 267-272. 1997.

[4] R. Kusner and N. Schmitt: The spinor representation of surfaces in space, (dg-ga/9610005).

[5] K. Moriya: On a variety of algebraic minimal surfaces in Euclidean 4-space, Tokyo J. Math. 21 (1998), 121-134.

[6] K. Moriya: On a variety of Weierstrass data for branched minimal surfaces in Euclidean space, in Differential geometry and applications (Brno, 1998), 207-213, Masaryk Univ., Brno, 1999.

[7] R. Osserman: Global properties of minimal surfaces in $E^{3}$ and $E^{n}$, Ann. Math. 80 (1964), 340-364.

[8] J. Pérez and A. Ros: The space of properly embedded minimal surfaces with finite total curvature, Indiana Univ. Math. J. 45 (1996), 177-204.

[9] J. Pérez and A. Ros: The space of complete minimal surfaces with finite total curvature as Lagrangian submanifold, Trans. Amer. Math. Soc. 351 (1999), 3935-3952.

[10] G.P. Pirola: Algebraic curves and non-rigid minimal surfaces in the Euclidean space, Pacific J. Math. 183 (1998), 333-357.

[11] G.P. Pirola: The infinitesimal variation of the spin abelian differentials and periodic minimal surfaces, Comm. Anal. Geom. 6 (1998), 393-426.

[12] A. Ros: Compactness of spaces of properly embedded minimal surfaces with finite total curvature, Indiana Univ. Math. J. 44 (1995), 139-152.

[13] K. Yang: Complete Minimal Surfaces of Finite Total Curvature, Kluwer Academic Publishers Group, Dordrecht, 1994.

Institute of Mathematics

University of Tsukuba

Tsukuba-shi

Ibaraki 305-8571 Japan

e-mail: moriya@math.tsukuba.ac.jp 